\title{
DICCIONARIO COMBINATORIO PARA HABLANTES DE PORTUGUÉS: PRINCIPIOS METODOLÓGICOS
}

\section{COMBINATORY DICTIONARY FOR PORTUGUESE SPEAKERS: METHODOLOGICAL PRINCIPLES}

\author{
Rebeca Martínez Aguirre \\ Universidad de Alcalá \\ rebeca.martineza@.edu.uah.es
}

\begin{abstract}
RESUMEN
El presente trabajo tiene como principal objetivo exponer la metodología planteada para la elaboración de un modelo de diccionario combinatorio dirigido a aprendices de español que tienen el portugués como lengua materna y adscrito a los niveles B1 y B2 del Marco Común Europeo de Referencia para las lenguas. Con este proyecto, enmarcado dentro de los límites académicos de la realización de una tesis doctoral, se pretende crear un prototipo de herramienta didáctica que ayude al hablante lusófono en el desarrollo y la consolidación de su conocimiento combinatorio y colocacional. Dicha propuesta responde a tres factores fundamentales. En primer lugar, la creciente importancia que, gracias a la influencia ejercida por el Enfoque Léxico, se ha concedido en las últimas décadas a la enseñanza de las colocaciones y otros cbunks o secuencias lingüísticas. En segundo lugar, a la acusada falta de sistematicidad con la que estas unidades han sido abordadas en los repertorios lexicográficos de la lengua española. Y en tercer lugar, la evidente carencia de herramientas combinatorias bilingües con las que se topan los estudiantes de español lengua extranjera. Proponemos así la creación de un diccionario combinatorio conformado a partir de un corpus de colocaciones y combinaciones léxicas estables, y cuyos fundamentos de macro y microestructura expondremos a continuación.
\end{abstract}

Palabras clave: colocación léxica, lexicografía bilingüe, diccionario combinatorio

\begin{abstract}
The main goal of the present work is to show the methodology used to create a model of combinatory dictionary aimed at Spanish as foreign language students who have the Portuguese as their native language. This dictionary is developed according to the guidelines of the B1 and B2 levels of the Common European Framework of Reference for Languages. This project is framed inside the academic limits of a $\mathrm{PhD}$ and attempt to create a prototype of didactical tool in order to help the Portuguese-speaking to consolidate and improve their combinatory and collocational knowledge. The present proposal reflects the needs of three main factors. First of all, the increasing profile of teaching collocations and other chunks, largely due to the influence exerted by the lexical approach. Secondly, the lack of lexicographical repertories where this chunks has been properly worked. Finally, the obvious lack of bilingual and combinatory tools provided to the Spanish as foreign language students. Therefore, we will present below the micro and macrostructural features of a combinatory dictionary proposal created in the basis of a corpus of collocations and other steady chunks.
\end{abstract}

Keywords: lexical collocation; bilingual lexicography; combinatory dictionary 


\section{CONSIDERACIONES PREVIAS}

La influencia ejercida en los últimos años por los principios postulados por el Enfoque Léxico ha situado las colocaciones en el epicentro del debate lingüístico y didáctico. Tradicionalmente el léxico ha constituido uno de los pilares fundamentales en la enseñanza y aprendizaje de lenguas extranjeras. Si el dominio de los mecanismos gramaticales resulta fundamental para garantizar una producción lingüística satisfactoria, no es menos importante el conocimiento de las unidades léxicas y sus posibilidades de combinación puesto que, como señalan Higueras García (2004, p. 481; 2007, p. 17) y Pérez Basanta (1995 ap. Alvar Ezquerra, 2003, p. 11), aprender una palabra es mucho más que reconocer y memorizar su forma gráfica, su pronunciación, su significado y sus usos gramaticales, aprender una palabra supone, además, conocer sus posibilidades y restricciones de combinación con otras unidades. Y es que de poco le servirá a un aprendiz de español conocer la voz paseo si no sabe que para utilizarla correctamente debe construirla con el verbo dar y no con hacer, por ejemplo, como sucede con excursión.

El reconocimiento de la importancia de la enseñanza de las colocaciones y de otros bloques o secuencias lingüísticas se remonta a los orígenes de la propia noción de colocación ya que fue Palmer (1933) - precursor del mismo ${ }^{1}$ - quien, de forma paralela a su definición lingüística, aportó una visión más pedagógica del concepto al subrayar las dificultades de aprendizaje que representaban las "sucesiones de palabras", motivo por el cual estimaba necesario un enfoque específico para dominarlas (Palmer, 1933 ap. Cowie, 2002 [1999], p. 54). Años después, también Nation (1990, p. 32) se preocupó por la combinatoria léxica y su implicación en el aprendizaje del léxico, y reconoció que "knowing a word involves having some expectation of the words that it will collocate with" ["conocer una palabra implica tener ciertas expectativas sobre las palabras con las que esta coocurrirá’]. El autor admite la importancia de las colocaciones por constituir la base del

\footnotetext{
${ }^{1}$ Aunque suele atribuirse al sistemista británico Firth (1957) la introducción del término collocation en los estudios lingüísticos para referirse a la coocurrencia habitual de unidades léxicas (Alonso Ramos, 19941995, p. 9; Corpas Pastor, 1996, p. 55; Koike, 2001, p. 16), autores como Mitchell (1971, p. 35) y Nesselhauf (2004, p. 2) adjudican su origen a Harold E. Palmer (1933) quien, ya anteriormente, lo había contemplado como "a succession of two or more words that must be learnt as an integral whole and not pieced together from its constituents parts" ["una sucesión de dos o más palabras que deben aprenderse como un todo y no reconstruirse a partir de sus partes constituyentes”] (Palmer, 1933 ap. Cowie 2002 [1999], p. 54).
} 
aprendizaje, el conocimiento y el uso de la lengua (2001, p. 321), y reclama la necesidad de investigar en este campo para:

a) conocer cuáles son las colocaciones de alta frecuencia y de estas cuáles son impredecibles,

b) saber cuáles son los patrones colocacionales más comunes, y

c) crear diccionarios que ayuden a los estudiantes a enfrentarse a las colocaciones de baja frecuencia (2001, p. 328).

Pero el auténtico impulsor de la enseñanza de las colocaciones y la combinatoria léxica fue Michael Lewis (1993), padre del Enfoque Léxico. El Enfoque Léxico surge en el seno del Enfoque Comunicativo, adoptando los fundamentos en él enunciados y añadiendo como eje fundamental el papel del léxico como principio organizador del contenido y la metodología de la enseñanza de lenguas extranjeras. Según Lewis (1993, p. 51), el principal objetivo de la lengua es la creación y el intercambio de significado, y, en dicho proceso, la implicación del léxico es mayor que la de las estructuras gramaticales. En consecuencia, una focalización en la comunicación conlleva, necesariamente, una mayor concentración en el vocabulario y un menor énfasis en la gramática (1993, p. 33). Para Lewis (1993, p. 51), "language consists of grammaticalised lexis, not lexicalised grammar" ["la lengua consiste en léxico gramaticalizado, no en gramática lexicalizada"], es decir, que el autor concibe la lengua no como un conjunto de patrones gramaticales con casillas vacías que deben rellenarse con unidades léxicas sino como un conjunto de chunks o unidades léxicas multipalabra que se relacionan entre sí mediante estructuras sintácticas. De esta forma, el léxico se convierte no solo en el pilar básico de la lengua sino también de su aprendizaje, invirtiendo la tradicional postura estructuralista que abogaba por un dominio de las reglas gramaticales del sistema como conocimiento facilitador de oraciones. En su lugar, son las unidades multipalabra las que permiten al aprendiz detectar los patrones morfosintácticos de la lengua y lograr el dominio del sistema sintáctico (1993, p. 95).

\section{COLOCACIONES Y DICCIONARIOS}

Es de sobra conocido el valor didáctico que tradicionalmente se le ha atribuido al diccionario en el proceso de adquisición de lenguas tanto maternas como extranjeras 
(Alvar Ezquerra, 2001, 2003; Martínez Navarro, 2005; Azorín Fernández y Martínez Egido, 2010; Perdiguero Villarreal, 2010). En dicho proceso, el profesor debe enseñar al alumno a valorar y emplear la lengua, y para ello debe proporcionarle una serie de herramientas y fuentes de información que le permitan acceder a conocimientos por sí mismo, herramientas de entre las cuales el diccionario constituye el instrumento más útil y provechoso (Prado Aragonés, 2005a, p. 19; 2005b, pp. 208-209).

No son pocos los trabajos que en los últimos tiempos han centrado su atención en el tratamiento recibido por las colocaciones en los distintos diccionarios que los aprendices extranjeros de español tienen a su disposición y si existe un punto en común a todos ellos, podemos decir que es la desatención y la falta de sistematicidad generalmente observadas ${ }^{2}$. Son escasas las combinaciones incluidas en los repertorios y en el caso de aquellas que sí están recogidas, la ausencia de un tratamiento exhaustivo en su registro por parte de los lexicógrafos resulta evidente. Existen vacilaciones en su denominación, falta uniformidad en su representación tipográfica y no hay acuerdo en cuanto a la entrada que sirve de acogida y la parte del artículo en la que se presentan. Todas estas incongruencias y lagunas de información no hacen sino dificultar las consultas de los usuarios, algo que ha llevado a los autores no solo a poner en duda la utilidad real de estos diccionarios para la producción y comprensión de las colocaciones, sino además a poner de relieve la necesidad de crear repertorios lexicográficos específicos con los que proveer al alumnado de una herramienta que le ayude en el desarrollo de su conocimiento combinatorio y colocacional (Castillo Carballo, 2004, 2015; Higueras García, 2004; Martínez Navarro, 2005; Romero Aguilera, 2008; Azorín Fernández y Martínez Egido, 2010; Molina Díaz 2010, entre otros).

Como es bien sabido, en el ámbito monolingüe, actualmente contamos ya con repertorios de importante peso como el Diccionario de colocaciones del españo $\beta$ — dirigido

\footnotetext{
2 Sobre la inclusión de las colocaciones en los diccionarios de lengua española vid. Castillo Carballo (2004), Ruiz Martínez (2007), Romero Aguilera (2008), Molina Díaz (2010), Alba Quiñones (2014), Corpas Pastor (2017), Maldonado González (2017) y Martínez Aguirre (2017), entre otros.

${ }^{3} \mathrm{El} \mathrm{DiCE}$ se acoge a los preceptos establecidos por la Lexicología explicativa y combinatoria de Mel'čuk et al. (1995). A propósito de sus características y contenido vid. Alonso Ramos (2010).
} 
por Margarita Alonso Ramos (2004)—, y los Diccionarios combinatorios del español contemporáneo REDES y Práctico ${ }^{4}$-ambos dirigidos por Ignacio Bosque Muñoz (2004, 2006)-. Cabe señalar que si bien es cierto que ninguno de ellos está explícitamente dirigido a hablantes no nativos, también lo es que los tres pueden servir de ayuda a aprendices que ya cuentan con cierto nivel de competencia lingüística.

En el ámbito bilingüe, por el contrario, no disponemos, hasta el momento, de ningún repertorio publicado y únicamente podemos aludir a un proyecto que actualmente se encuentran en fase de desarrollo. Nos referimos al Diccionario combinatorio españolitaliano, un repertorio enfocado a las posibilidades combinatorias de las palabras que está siendo creado en solitario, por el profesor Simone Greco de la Universidad de Bari. Asimismo, cabe hacer referencia a otros dos proyectos propuestos recientemente. El primero de ellos corresponde al Diccionario de combinaciones léxicas para la enseñanza-aprendizaje de E/LE por niveles, presentado en el año 2012 a modo de tesis doctoral por Verónica Ferrando Aramo (Universidad Rovira y Virgili) y el segundo remite al Dicionário Combinatório on-line, una propuesta coordinada por la profesora Enilde Faulstich de la Universidad de Brasilia entre los años 2011 y $2014^{5}$.

A la vista de esta carencia de herramientas lexicográficas combinatorias y también en el marco de realización de una tesis doctoral, surge esta propuesta de creación de un modelo de diccionario combinatorio destinado a hablantes de portugués. Seguidamente, procederemos a exponer la metodología de elaboración planteada que actualmente se encuentra en vías de desarrollo- sobre la que se asienta su elaboración.

\section{DICCIONARIO COMBINATORIO ESPAÑOL-PORTUGUÉS: METODOLOGÍA DE ELABORACIÓN}

El modelo de diccionario que planteamos se caracteriza, tal como ya adelantábamos en líneas precedentes, por constituir un repertorio combinatorio dirigido a hablantes que tienen el portugués como lengua materna y por adscribirse a los niveles B1 y B2

\footnotetext{
${ }^{4}$ Sobre los principios de combinatoria léxica que subyacen a los diccionarios REDES y Práctico, V. Bosque Muñoz (2004 y 2006).

${ }^{5}$ Hasta donde tenemos conocimiento, ninguno de los dos proyectos tiene continuación en la actualidad.
} 
del Marco Común Europeo de Referencia para las lenguas. Está eminentemente concebido como un instrumento didáctico destinado a estudiantes y profesores de español como lengua extranjera, no obstante, podría servir además como herramienta lingüística de apoyo a otros profesionales de la lengua como traductores o correctores bilingües.

Actualmente el desarrollo de la propuesta se encuentra en una fase inicial de elaboración en la que se está procediendo a la preparación y organización de los materiales así como al establecimiento de las bases sobre las que se fundamentarán la macro y la microestructura.

\subsection{MARCO TEÓRICO Y CONTENIDO}

Tal y como señalábamos anteriormente, el punto de partida de la propuesta aquí planteada remite al concepto de colocación léxica y su papel en la enseñanza del español como lengua extranjera. Es de sobra conocido el controvertido debate teórico que en los últimos tiempos se viene produciendo en torno a dicho concepto. Son muchos los autores que, desde diferentes perspectivas, han tratado y tratan de delimitar las características definitorias de estas combinaciones de palabras así como de situarlas en una u otra parcela lingüística, y, por el momento, es evidente que no hay visos de alcanzar unanimidad al respecto. De hecho, como señala Nesselhauf (2004, p. 1), ante la cuestión ¿qué son las colocaciones? posiblemente podríamos llegar a hablar de tantas respuestas como autores existen sobre el tema.

En el caso concreto que aquí nos ocupa, nuestra postura se adscribe a los fundamentos establecidos por Ignacio Bosque (2001a, 2011), quien adscribe las colocaciones a la interfaz léxico-sintaxis. Según sostiene el autor, las colocaciones constituyen manifestaciones de selección léxica en las que el predicado selecciona a sus argumentos restringiendo el conjunto de unidades que estos pueden denotar conforme a una serie de rasgos semánticos que ambos tienen en común. En el caso de verbos y sustantivos, esta restricción se produce sobre sus argumentos internos, mientras que en el de adjetivos y adverbios, la restricción recae sobre sus argumentos externos (Bosque Muñoz, 2017, p. 10). 
En contra de lo que algunos autores opinan ${ }^{6}$, desde su punto de vista, las combinaciones originadas por restricciones selectivas deben quedar excluidas del marco de las colocaciones puesto que, en la mayor parte de los casos, estas no aportan información de naturaleza lingüística sino extralingüística (Bosque Muñoz, 2001b, p. 39; 2004a, pp. XCVII-C; 2004b, pp. 25-27) ${ }^{7}$. De este modo, unidades como planear una gaviota o un avión no deben considerarse colocaciones pues no aportan nada sobre el conocimiento del idioma sino sobre los seres o las cosas que tienen la capacidad de planear de manera que para construir esta lista de combinaciones "no hay que analizar palabras; basta con mirar alrededor" (Bosque Muñoz, 2004a, p. XCVIII). En consecuencia, el autor habla de colocaciones en el caso de combinaciones como planear las dudas, las sospechas o los miedos, en las que la relación predicado-argumento se restringe mediante informaciones semánticas objetivas que forman parte del idioma, siendo necesario un conocimiento específico del mismo para poder construirlas. Así, en su producción, en lugar de examinar el mundo que nos rodea, deben analizarse "la estructura del idioma y las clases lingüísticas que se reconocen en él” (Bosque Muñoz, 2004a, XCIX). Pongamos por caso el verbo derrumbarse, predicado que selecciona dos paradigmas de argumentos diferentes. El primero — el físico— puede conformarse a partir de la definición del verbo y de la observación de la realidad: derrumbarse un rascacielos, una pared, una muralla, etc. El segundo - el figurado- debe aprenderse específicamente: derrumbarse las esperanzas, los sueños, las expectativas, etc. Es más, si deseamos traducir ambos paradigmas a otra lengua, mientras la traducción literal del verbo proporcionará prácticamente el mismo paradigma físico, en el caso del figurado no tiene por qué existir una coincidencia exacta de equivalentes pues los paradigmas de colocaciones son propios e idiosincrásicos de cada idioma (Bosque Muñoz, 2004a, p. CI).

De acuerdo con este tipo de selección, para Bosque Muñoz, en oposición a lo expuesto por otros estudiosos, no es la base la que selecciona el colocativo con el que se combina sino a la inversa, es el colocativo o predicado el que selecciona la base o

\footnotetext{
${ }^{6}$ Cfr. Corpas Pastor (1996), Koike (2001), Alonso Ramos (2010).

7 Las restricciones selectivas dan cuenta de rasgos semánticos del tipo 'humano', 'animado', 'concreto', 'objeto material', 'artefacto', etc. (Bosque Muñoz 2004a, XCVII; 2004b, p. 25).
} 
$\operatorname{argumento}^{8}$. Además, tal como demuestra el autor, las unidades seleccionadas no son piezas aisladas sino clases léxicas de mayor o menor intensión de modo que, en la mayor parte de los casos, la relación entre base y colocativo no es biunívoca, es decir, no constituyen casos únicos de compatibilidad semántica (Bosque Muñoz, 2001a, p. 10; 2011, p. VIII). Así, la selección que el adjetivo acérrimo hace del sustantivo enemigo no es exclusiva, tal y como la mayor parte de la literatura precedente ha interpretado. Por el contrario, lo cierto es que el mencionado adjetivo selecciona toda una clase de sustantivos, la de aquellos que denotan "personas enfrentadas a algo o a alguien", entre los que se encuentra, efectivamente, la forma enemigo, pero también adversario, detractor o rival, entre otros. Pero no solo eso, sino que además selecciona otras clases como la de los sustantivos que designan "al que apoya o defiende algo o a alguien, muy frecuentemente ideas, posturas, acciones o instituciones", entre los que se encuentran defensor, seguidor o partidario; o la de aquellos que denotan "al partidario o defensor de las personas, movimientos, instituciones o ideas políticas, religiosas o deportivas que se asocian con el nombre o el adjetivo del que se derivan", como nacionalista, comunista o falangista, entre otras (2001a, p. 15). Por este motivo, Bosque Muñoz (2011, p. IX) defiende que las Funciones Léxicas propuestas por la Teoría Sentido Texto ${ }^{9}$ deberían ser formuladas a partir de clases léxicas y no de unidades concretas pues esto permitiría

\footnotetext{
${ }^{8}$ Para más información sobre las restricciones ejercidas por los predicados sobre sus argumentos vid. Bosque Muñoz (2004b, pp. 23-31).

${ }^{9}$ La Teoría Sentido-Texto fue propuesta por Mel’čuk y Zholkovsky en el año 1970. Se trata de "una teoría lexicográfica vinculada a la lingüística general y relacionada con la gramática de dependencias" (Barrios Rodríguez 2006, p. 704). Fue aplicada por Mel'čuk y sus colaboradores, entre 1984 y 1999, en la elaboración del Dictionnaire Explicatif et Combinatoire du français contemporain cuyos principales objetivos son ofrecer una explicación semántica formal (explicatif) y exponer la combinatoria léxica (combinatoire) de cada entrada contenida (Mel'čuk et al., 1995: p. 10). Dentro de su marco teórico las colocaciones son explicadas mediante Funciones Léxicas. Según Mel'čuk (1995, p. 188) una Función Léxica (en adelante FL) f es una función que asocia a unidad léxica dada L - argumento o palabra llave de $\mathrm{f}$ - un conjunto más o menos amplio de elementos léxicos sinónimos $\{\mathrm{Li}\}$ — valor de $\mathrm{f}$ - que pueden ser seleccionados por L para expresar un significado que se corresponde con $\mathrm{f}$. Esta relación se representa mediante la fórmula $\mathrm{f}(\mathrm{x})=\mathrm{y}$. Como se desprende de su definición, la palabra llave es el lexema sobre el que se aplica la FL y el valor de la FL es el lexema que expresa el sentido de la FL: Función (palabra llave) = valor de FL. Así, por ejemplo, en Magn (to condemn) = strongly, la FL Magn representa el sentido "intensamente", to condemn es la palabra llave (o base) y strongly el valor de la FL (o colocativo).
} 
al hablante establecer generalizaciones y deducir por sí mismo nuevas colocaciones en lugar de tener que aprender combinaciones aisladas ${ }^{10}$.

Ahora bien, teniendo en cuenta que nuestro objetivo es crear un diccionario destinado a hablantes extranjeros y que se trata de una herramienta inherentemente didáctica, coincidimos con Higueras García (2017, p. 252) al contemplar que esta consideración tan restringida del término nos obligaría a prescindir de numerosas combinaciones que, a pesar de no ser producto de restricciones intralingüísticas, pueden resultar de gran utilidad para el usuario por constituir combinaciones léxicas estables que, en algunos casos, pueden dar lugar a problemas de producción. De esta forma, hemos resuelto que el cuerpo del repertorio esté constituido tanto por colocaciones — cortarse la comunicación, ataque de ansiedad, saludar calurosamente - como por otras combinaciones con carácter estable ${ }^{11}$ —aprobar un examen, fiebre alta, citar textualmente-.

El corpus compilado hasta el momento para la creación del Diccionario combinatorio español-portugués está integrado por un total de 633 unidades, de las cuales 148 pertenecen al nivel B1 y 485 al nivel B2. Se trata de un corpus germinal, concebido como un repertorio susceptible de ser ampliado y modificado posteriormente con nuevas unidades. Todas las combinaciones que lo integran han sido extraídas de los catálogos de Nociones Generales y Nociones Específicas del Plan Curricular del Instituto Cervantes (PCIC) (2007 [2006]) correspondientes a los niveles mencionados. En un primer momento, se determinó que además este corpus fuera enriquecido con otras unidades tomadas de diversos materiales didácticos como manuales, repertorios léxicos y el diccionario combinatorio REDES (Bosque Muñoz, 2004a) pues, tal y como se indica expresamente en el PCIC, sus inventarios deben considerarse abiertos "en cuanto que siempre podrían añadirse nuevos elementos, tanto en sentido horizontal

\footnotetext{
${ }^{10}$ Frente a estos paradigmas de colocaciones deducibles, el autor también reconoce la existencia de unidades no deducibles en las que o bien los colocativos solo coocurren con una base - conciliar el sueño- o bien se combinan con paradigmas cerrados y reducidos de bases - fruncir el ceño, el entrecejo, las cejas- (Bosque Muñoz, 2011, p. IX).

${ }^{11}$ En su primera fundamentación del concepto de colocación, Higueras García (2007, pp. 39-40) acuñó el término de colocaciones no prototípicas para referirse a este conjunto de combinaciones de palabras frecuentes y estables.
} 
(completando las series), como en sentido vertical (con nuevos elementos en cada nivel)" (2007 [2006], pp. 393-394). Sin embargo, dada la elevada suma de combinaciones obtenida tras el vaciado de la obra, y teniendo en cuenta el calibre del trabajo académico en el que esta elaboración se enmarca, se ha decidido limitar la selección al conjunto señalado ${ }^{12}$.

\subsection{Principios de macroestructura}

Uno de los aspectos más observados en los estudios que versan sobre la presencia de las colocaciones en los diccionarios, es la entrada que da cabida a la información combinatoria pues, como advierte Castillo Carballo (2002, p. 99), "la función lexicográfica variará dependiendo de dónde aparezca la información, porque si se ubica en la base se contribuirá a la producción, mientras que si se halla en el colocativo se facilitará la recepción lingüística". Esto se debe a que, por lo general, el hablante no nativo conoce la palabra que funciona como base de la colocación y necesita el colocativo, es decir, aquella con que debe combinarla. De esta forma, su única forma de acceder a la combinación y efectuar correctamente la codificación será acudir al artículo lexicográfico de la base. De forma paralela, en el proceso de descodificación y ante el desconocimiento del significado de la unidad que funciona como colocativo, el aprendiz buscará solventar sus dudas sobre el mismo acudiendo a la entrada que a este corresponde (Calderón Campos, 1994, pp. 80-81). Puesto que el principal objetivo del modelo diseñado es servir de apoyo al hablante extranjero tanto en la codificación como en la descodificación, el índice lemático del repertorio será construido a partir de las bases y los colocativos de las colocaciones así como de los dos elementos que integran las combinaciones estables. En consecuencia, la macroestructura estará conformada por un elenco de sustantivos, adjetivos, verbos y adverbios dispuestos alfabéticamente.

\footnotetext{
12 Cabe señalar, no obstante, que el esbozo que se presentará en la tesis doctoral no contendrá la totalidad del corpus compendiado sino únicamente un conjunto limitado de unidades que resulte lo suficientemente representativo como para ilustrar el modelo de diccionario planificado. En este sentido, la selección de combinaciones se hará atendiendo a los diferentes tipos de estructuras combinatorias recopiladas.
} 
Por otra parte, se ha convenido establecer asimismo un segundo índice en el que todas las combinaciones se encuentren ordenadas bajo los descriptores que integran las Nociones Generales y Específicas del PCIC, esto es, a partir del índice de temas "entre los que se distribuye el conjunto de las entradas del inventario" (Instituto Cervantes, 2007 [2006], pp. 445). Esta clasificación nos parece que puede resultar de especial interés para profesores y para estudiantes pues en ella se podrá consultar tanto el nivel al que pertenece cada combinación como el conjunto de unidades asociadas a un mismo tema.

$\left.\begin{array}{|l}\text { 6. EDUCACIÓN } \\ \text { 6.3. SISTEMA EDUCATIVO } \\ \text { - Clase térica } \\ \text { - Pedir una beca } \\ \text { - Formación continua } \\ \text { - Educación universitaria } \\ \text { - Educación superior } \\ \text { - Educación pública } \\ \text { - Educación privada } \\ \text { - Educación religiosa } \\ \text { - Educación laica } \\ \text { - Ofrecer educación } \\ \text { - Recibir educación } \\ \text { - Solicitar una beca } \\ \text { - Conceder una beca } \\ \text { - Disfrutar de una beca } \\ \text { - Obtener una beca } \\ \text { 6.4. APRENDIZAJE Y ENSEÑANZA } \\ \text { - Saber de memoria } \\ \text { - Aprender de memoria } \\ \text { - Estudia de memoria } \\ \text { - Alumno brillante } \\ \text { - Sacar conclusiones } \\ \text { - Recibir clases } \\ \text { 6.5. EXÁMENES Y CALIFICACIONES } \\ \text { - Hacer un examen oral } \\ \text { - Aprobar un examen oral } \\ \text { - Suspender un examen oral } \\ \text { - Aprobar el curso } \\ \text { - Examen parcial } \\ \text { - Examen final } \\ \text { - Evaluación parcial } \\ \text { - Evaluación final }\end{array}\right\} \mathrm{B}$




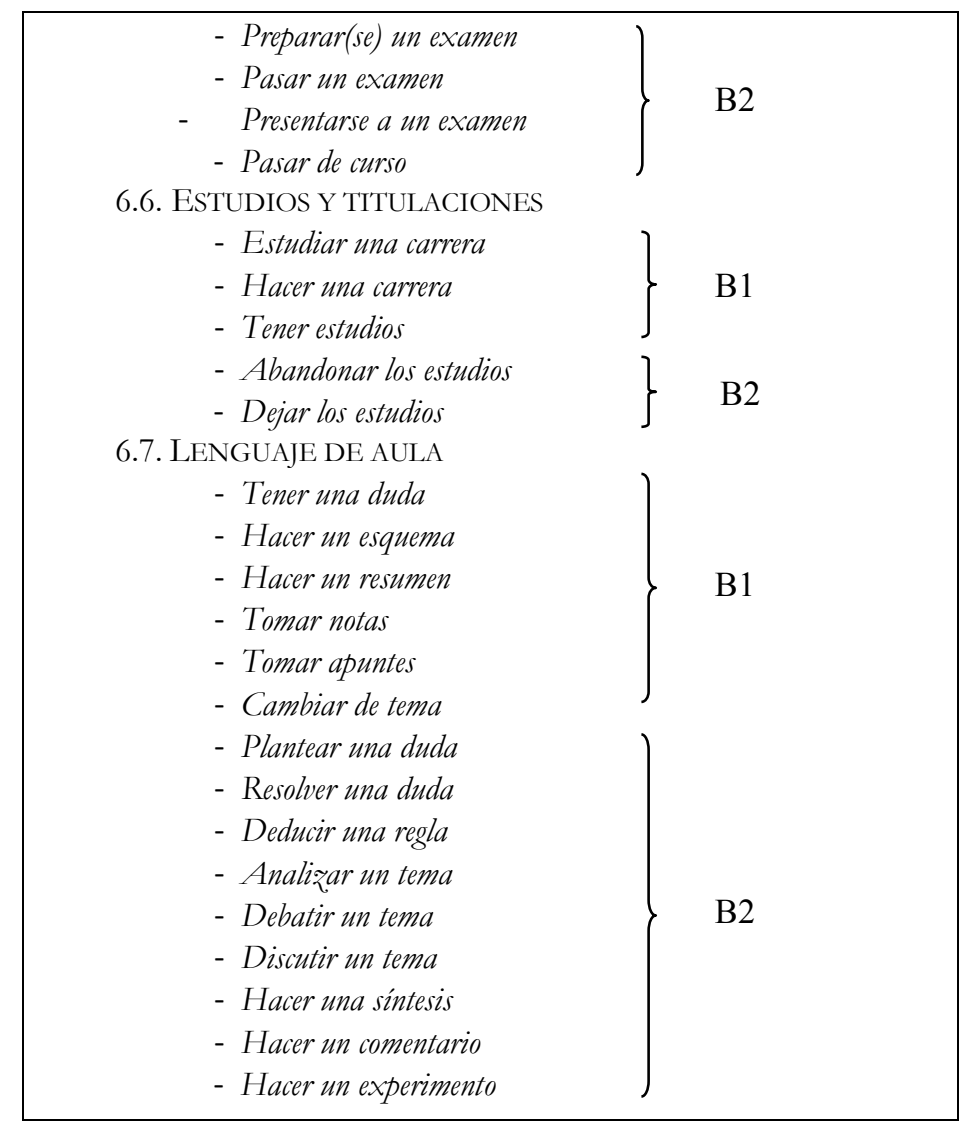

Figura 1. Propuesta de índice onomasiológico, descriptor 6. Educación

\subsection{Principios de microestructura}

El modelo lexicográfico que planteamos se enmarca dentro de la tipología de diccionarios semibilingües, los cuales se encuentran a medio camino entre los monolingües y los bilingües, englobando información propia de los primeros y de los segundos. La lexicografía semibilingüe constituye una rama teórica todavía en proceso de desarrollo debido precisamente a su naturaleza híbrida ${ }^{13}$, sin embargo, nos parece la más adecuada para nuestra propuesta por aprovechar y aunar las ventajas pedagógicas de ambas vertientes. La estructura prototípica que conforma el diccionario semibilingüe está integrada por "lema en L2 + definición en L2 + equivalente en L1"

\footnotetext{
${ }^{13}$ Como expone Climent de Benito (2008, p. 417), "el concepto de diccionario semibilingüe se encuentra poco estudiado en la tradición lexicográfica española y, por ello, mal clasificado, especialmente debido a dos factores: por un lado, por su naturaleza compleja entre monolingüe y bilingüe; por otro, por la confusión terminológica existente, pues, entre otras, se recurre a diversas denominaciones para la misma entidad (diccionario bibrido, bridge dictionary, bilingualizado...)".
} 
(Climent de Benito, 2008, p. 418), no obstante, en el modelo que planteamos prescindiremos de la definición en L2 y nos limitaremos a la inclusión del equivalente en L1. Asimismo se tomarán otros datos propios de los repertorios monolingües como son los ejemplos y las informaciones complementarias. De este modo, lengua materna y lengua meta se coordinan en la equivalencia, ejemplificación y contextualización de las combinaciones de palabras idiosincrásicas de la lengua extranjera, todo ello con el objetivo de facilitar que el usuario saque el máximo rendimiento a la información lingüística ofrecida.

Para la disposición de la microestructura, hemos tomado en consideración los preceptos establecidos en obras ya consolidadas como el Diccionario de colocaciones del español (Alonso Ramos, 2004) y el OXFORD Collocations Dictionary —disponible para consulta en línea ${ }^{14}$ - Las pautas de organización del artículo lexicográfico que por el momento hemos establecido son las siguientes:

i. En primer lugar se sitúa el lema, acompañado mediante abreviatura de su categoría gramatical que, en el caso de los sustantivos, se complementa con el género.

\section{Calor, sust. masc.}

Figura 2. artículo lexicográfico del lema calor (I).

ii. Bajo el lema, aparecerán ordenados numéricamente unos discriminadores semánticos que esbocen sus diferentes acepciones, distinguiendo, así, los significados presentados en el contexto de cada combinación o conjunto de combinaciones. Dichos discriminadores se ofrecerán en español y en portugués.

\section{Calor, sust. masc. \\ 1 sensación, sensação}

Figura 3. artículo lexicográfico del lema calor (II).

iii. Tras el discriminador semántico, se indicarán las colocaciones y combinaciones estables en las que el lema participa.

${ }^{14}$ Recurso en http://www.freecollocation.com/ 
Todas las combinaciones se agruparán indistintamente atendiendo a su estructura morfosintáctica, la cual será indicada al inicio de cada grupo. Para ello, seguiremos la clasificación propuesta por Koike (2001, p. 46) para las colocaciones: (1) sust + vb: sust $t_{\mathrm{Su}}+\mathrm{vb}, \mathrm{vb}+$ sust $_{\mathrm{CD}}, \mathrm{vb}+$ prep + sust; (2) sust + adj; (3) sust + prep $+\operatorname{sust}^{15}$; (4) vb + adv; (5) vb $+\operatorname{adj}^{16}$.

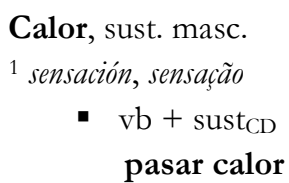

Figura 4. artículo lexicográfico del lema calor (III).

iv. Inmediatamente después de las combinaciones, se incluirán los equivalentes de las mismas, una etiqueta semántica a modo de glosa explicativa del significado y varios ejemplos de uso en español y portugués.

\section{Calor, sust. masc.}

${ }^{1}$ sensación, sensação

- $\mathbf{v b}+$ sust $_{\mathrm{CD}}$

pasar calor $\rightarrow$ passar calor [sentir, sentir]

- Pasé mucho calor durante el concierto = passei muito calor durante o concerto.

- Pasamos mucho calor esos días = passamos muito calor esses dias.

Figura 5. artículo lexicográfico del lema calor (IV).

En un primer momento, se pensó en extraer los equivalentes y ejemplos de uso del Diccionario Bilingüe de Uso español-portugués, portugués-español (Moreno y Maia, 2003) y, sobre todo, de los corpus paralelos Linguee y Glosbe puesto que en la mayor parte de los diccionarios bilingües no se incluye la combinatoria léxica. Sin embargo, una vez iniciada esta búsqueda, nos estamos topando con algunas dificultades ya que no en pocas ocasiones resulta costoso encontrar tanto el equivalente de la combinación como ejemplos válidos para los niveles predefinidos. Por este motivo, hemos

\footnotetext{
15 Aunque Koike (2001) emplea la nomenclatura sust + de + sust, nosotros hemos optado por utilizar sust + prep + sust para mantener la homogeneidad en todas las estructuras.

${ }^{16}$ Hemos omitido la estructura adv $+\mathrm{adj} /$ part porque el corpus no consta de ninguna colocación con ese esquema.
} 
SECCIÓN: LEXICOGRAFÍA

DICCIONARIO COMBINATORIO PARA

HABLANTES DE PORTUGUÉS

Rebeca Martínez Aguirre

decidido emplear otras herramientas lingüísticas como diccionarios monolingües y corpus del portugués.

v. Por último, se incluirán también informaciones complementarias de diversa índole sobre la combinación que puedan resultar de relevancia para el usuario en el empleo de la misma. Nos referimos a datos tales como observaciones sobre el uso o ausencia obligatoria de artículo, el régimen preposicional, el orden de los constituyentes, preferencias de uso, etc.

La inclusión de este tipo de informaciones remite a la noción defendida por la Lexicografía de aprendizaje, y más concretamente por la Teoría Funcional (Bergenholtz y Tarp, 2003), del diccionario como "herramienta concebida para satisfacer los tipos específicos de necesidades que tengan unos tipos específicos de usuarios en unos tipos específicos de situaciones sociales extra-lexicográficas" (Tarp, 2006, p. 297). Como es bien sabido, este enfoque aboga por una concepción de la lexicografía como disciplina científica independiente de la lingüística y por la creación del diccionario como un producto de utilidad hecho para satisfacer las necesidades humanas. De ahí que todas las consideraciones teóricas y prácticas deban basarse en la determinación de esas necesidades, es decir, en lo que se necesita para resolver los problemas específicos que le surgen a un grupo concreto de usuarios con unas características determinadas en unas situaciones de uso precisas (Bergenholtz y Tarp, 2003, p. 172) ${ }^{17}$.

Somos conscientes, por ende, de la importancia y la necesidad de definir sólida y fundamentadamente las necesidades particulares del hablante lusófono cara a la

${ }_{17}$ Bergenholtz y Tarp (2003, p. 173) determinan las siguientes propiedades como aquellas que se deben tomar en consideración para definir un grupo potencial de usuarios:

1. Which language is their mother tongue? [¿Qué idioma es su lengua materna?]

2. At what level do they master their mother tongue? [¿En qué nivel dominan su lengua materna?]

3. At what level do they master a foreign language? [¿En qué nivel dominan la lengua extranjera?]

4. How are their experience in translating between the languages in question? [¿Cuánta experiencia tienen en la traducción entre los idiomas en cuestión?

5. What is the level of their general cultural and encyclopaedic knowledge? [¿Cuál es el nivel de su conocimiento cultural y enciclopédico?]

6. At what level do they master the special subject field in question? [¿Qué nivel de dominio tienen del ámbito temático específico en cuestión?]

7. At what level do they master the corresponding LSP in their mother tongue? [¿Qué nivel de dominio tienen de la Lengua para Fines Específicos en su lengua materna?]

8. At what level do they master the corresponding LSP in the foreign language? [¿Qué nivel de dominio tienen de la Lengua para Fines Específicos en la lengua extranjera?] 
elaboración de una herramienta como la aquí descrita. Para ello, tendremos en cuenta las nueve categorías informativas establecidas por Bergenholtz y Tarp (2003, p. 175) ${ }^{18}$ :

1. Information about the native language.

2. Information about a foreign language.

3. Comparison between the native and a foreign language.

4. Information about culture and the world in general.

5. Information about the special subject field.

6. Comparison between the subject field in the native and foreign cultura.

7. Information about the native LSP.

8. Information about the foreign LSP.

9. Comparison between the native and foreign LSP.

Una vez expuesta la estructura diseñada para la creación de los artículos lexicográficos, presentamos, a continuación, a modo ilustrativo, la entrada completa correspondiente al lema calor.

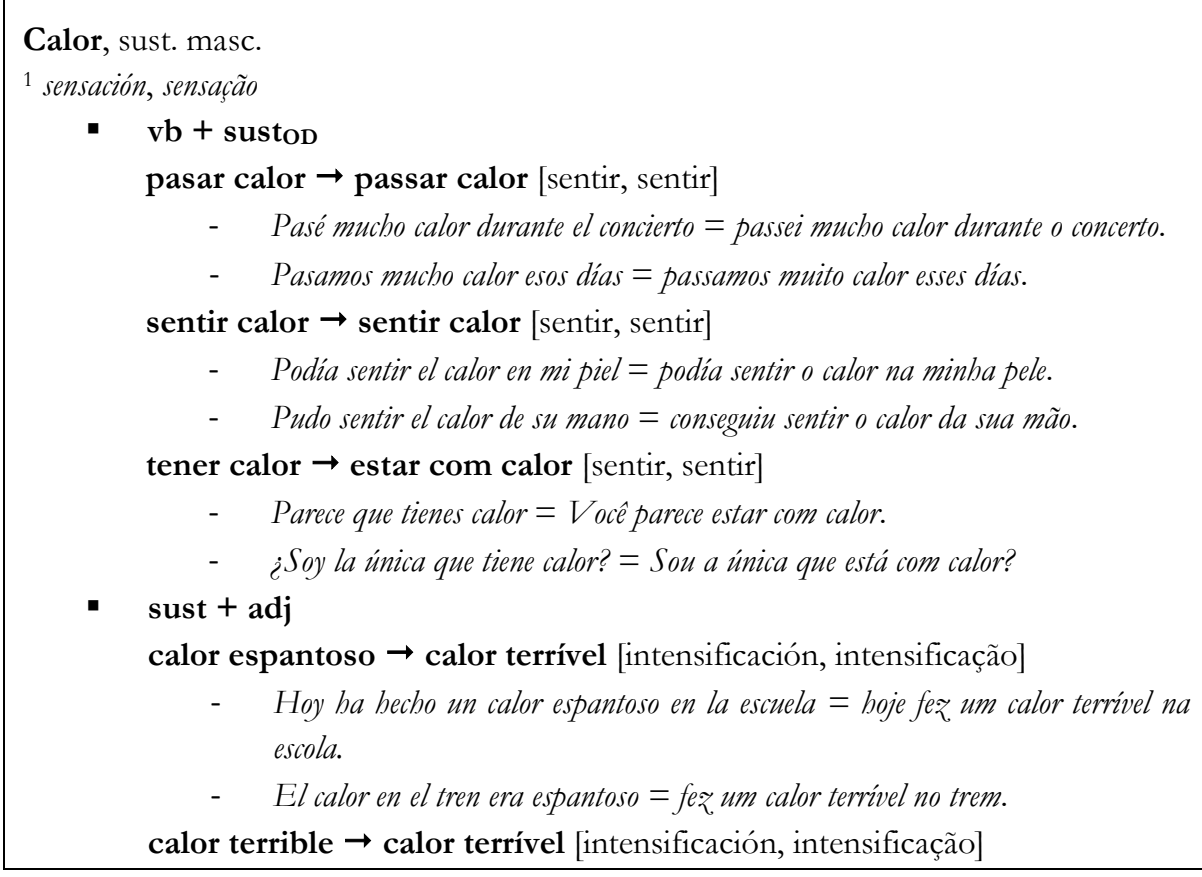

\footnotetext{
18 1. Información sobre la lengua nativa.

2. Información sobre la lengua extranjera.

3. Comparación entre el idioma nativo y un idioma extranjero.

4. Información sobre la cultura y el mundo en general.

5. Información sobre el ámbito temático específico.

6. Comparación entre el ámbito temático en la cultura nativa y extranjera.

7. Información sobre la Lengua para Fines Específicos nativa.

8. Información sobre la Lengua para Fines Específicos extranjera.

9. Comparación entre la Lengua para Fines Específicos nativa y extranjera.
} 


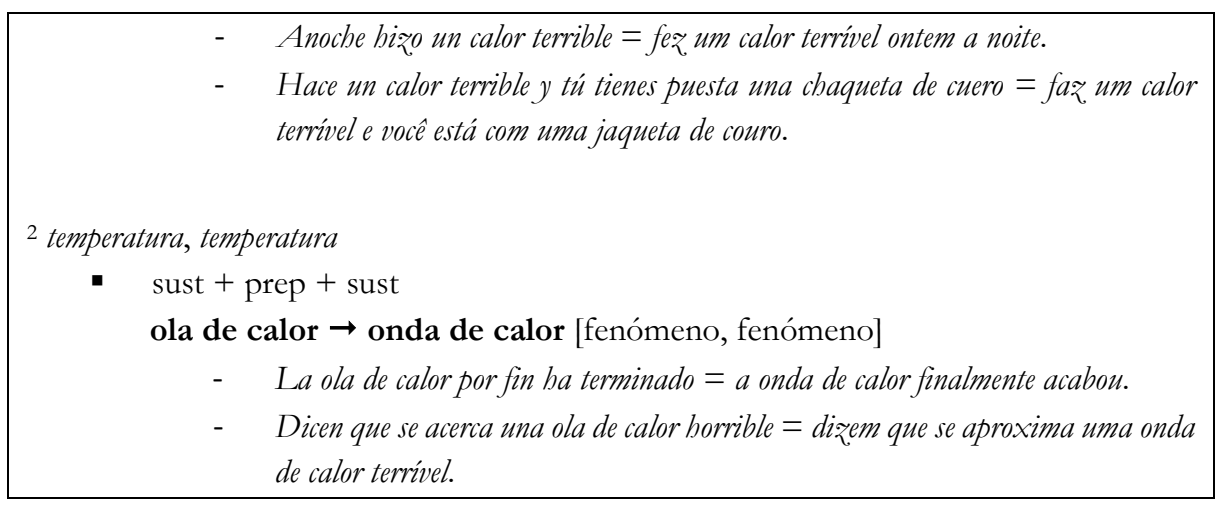

Figura 6. artículo lexicográfico del lema calor.

Como podemos observar, la entrada se inicia con el lema, su categoría gramatical y su género. Seguidamente se distinguen dos bloques diferenciados por los discriminadores semánticos o acepciones que el sustantivo presenta en las combinaciones recogidas. En el primero de ellos, calor ostenta el significado de 'sensación', mientras que en el segundo tiene el sentido de 'temperatura'. A su vez, el primer bloque se encuentra subdividido en otros dos subconjuntos. El primero de ellos responde a las combinaciones conformadas bajo la estructura verbo + sustantivo en función de objeto directo y el segundo a las integradas por el esquema sustantivo + adjetivo. Por otra parte, el bloque correspondiente al segundo discriminador semántico, únicamente cuenta con un segundo nivel de clasificación, el que abarca la estructura sustantivo + preposición + sustantivo. Por último, dentro de cada esqueleto morfosintáctico, se incluyen las combinaciones en las que participa el lema seguidas de sus respectivos equivalentes en portugués, una glosa explicativa del significado aproximado de la combinación y dos ejemplos de uso de la misma. Tal y como indicábamos anteriormente, tanto los discriminadores semánticos como las glosas y los ejemplos se presentan en español y en portugués, coordinando la lengua meta y la lengua materna del usuario.

\subsection{FORMATO DE LA OBRA}

En cuanto al formato del diccionario, lo más deseable sería poder acceder a un soporte electrónico que permitiera crear una herramienta dinámica y sin limitaciones en el almacenamiento de la información. Esto permitiría no solo registrar una más amplia y 
variada cantidad de datos lingüísticos, enlaces, imágenes — como redes de palabras-, etc. sino también más opciones de búsqueda, algo que en el formato tradicional está inevitablemente restringido. Sin embargo, es evidente que la carga de trabajo y programación que una elaboración de este tipo supone no se encuentra al alcance del marco de actuación de la propuesta que planteamos por lo que dicha posibilidad deberá quedar postergada y supeditada a la posibilidad de que, en un futuro, este proyecto pueda tener continuación.

\section{A MODO DE CONCLUSIÓN}

Tal y como hemos expuesto, las colocaciones léxicas ocupan un lugar fundamental en las investigaciones tanto de índole didáctica como lexicográfica, por constituir formas idiosincrásicas de la lengua cuyo conocimiento es necesario para que el discente logre alcanzar una correcta competencia comunicativa. No obstante, en la actualidad, todavía son escasos los proyectos que coordinan ambas corrientes. El modelo de diccionario cuya metodología de elaboración hemos mostrado en el presente trabajo tiene como objetivo fundamental servir de apoyo a los hablantes de portugués en el empleo y el aprendizaje de la combinatoria léxica del español. Tenemos plena consciencia de que se trata de una propuesta "ideal", en la que hemos buscado incluir todas aquellas informaciones que consideramos que resultarían de utilidad al destinatario potencial para el que la obra está diseñada. Con todo, sabemos también que, en caso de que dicho proyecto pudiera llevarse a cabo en el futuro, las directrices marcadas a priori posiblemente -por no decir con total seguridad- se verían modificadas por diversos condicionantes derivados del tratamiento "real" y específico de cada unidad léxica. Aun así, nuestro propósito es plantear una nueva línea de trabajo que contribuya, modestamente, al desarrollo actual de la lexicografía combinatoria bilingüe. 
SECCIÓN: LEXICOGRAFÍA

DICCIONARIO COMBINATORIO PARA

HABLANTES DE PORTUGUÉS

Rebeca Martínez Aguirre

\section{BIBLIOGRAFÍA}

Alba Quiñones, V. (2014). Sobre diccionarios monolingües de aprendizaje del español y colocaciones léxicas. Estudio e implicaciones didácticas, en M. ${ }^{a}$ P. Garcés. (Coord.), Lexicografía teórica y aplicada. A Coruña: Universidade da Coruña, pp. 119-134

Alonso Ramos, M. (1994-1995). Hacia una definición del concepto de colocación: de J. R. Firth a I. A. Mel'čuk, en Revista de lexicografia, 1, pp. 9-28.

Alonso Ramos, M. (2004). Diccionario de Colocaciones del Español, A Coruña: Universidade da Coruña. Recuperado de http://wwwdicesp.com/

Alonso Ramos, M. (2010). No importa si la llamas o no colocación, descríbela, en C. Mellado, P. Buján, C. Herrero, N. M. Iglesias y A. Mansilla. (Eds.), La fraseografia del S. XXI. Nuevas propuestas para el español y el alemán, Berlín: Frank \& Timme, pp. 55-80

Alvar Ezquerra, M. (2001). Los diccionarios y la enseñanza de la lengua, en M. C. Ayala. (Coord.), Diccionarios y enseñanz̧a, Alcalá de Henares: Universidad de Alcalá, pp. 13-30

Alvar Ezquerra, M. (2003). La enseñanza del léxico y el uso del diccionario, Madrid: Arco Libros.

Azorín Fernández, D. y Martínez Egido, J. J. (2010). Hacia una evaluación de las prestaciones didácticas del diccionario desde la perspectiva de los usuarios, en J. M. García y M. A. Castillo. (Coords.), Investigación lexicográfica para la enseñanza de lenguas, Málaga: Universidad de Málaga, pp. 51-67.

Barrios Rodríguez, M. a A. (2006). El aprendizaje de las colocaciones del españolCALLEX, una propuesta multimedia, en A. Álvarez et al. (Eds.), La competencia pragmática y la enseñanza del español como lengua extranjera, Oviedo, España: Servicio de Publicaciones de la Universidad de Oviedo, pp. 703-712.

Bergenholtz, H. y Tarp, S. (2003). Two opposing theories: On H.E. Wiegand's recent discovery of lexicographic functions. Hermes, Journal of Linguistics, 31, pp. 171-196.

Bosque Muñoz, I. (2001a). Sobre el concepto de 'colocación' y sus límites. Lingǘstica Española Actual. XXIII, 1, pp. 9-40.

Bosque Muñoz, I. (2001b). Sobre el concepto de 'lugar común’ desde el punto de vista gramatical. Pandora: revue d'etudes hispaniques, 1 , pp. 31-46.

Bosque Muñoz, I. (Dir.). (2004a). Redes. Diccionario combinatorio del español contemporáneo. Madrid: SM.

Bosque Muñoz, I. (2004b). La direccionalidad en los diccionarios combinatorios y el problema de la selección

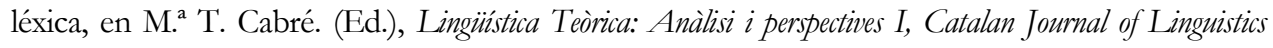
Monographies (pp. 13-58). Recuperado de http://filcat.uab.es/clt/publicacions/coleccions/monografies/LT-index2-propuesta.html

Bosque Muñoz, I. (Dir.) (2006). Diccionario combinatorio práctico del español contemporáneo, Madrid: SM. 
SECCIÓN: LEXICOGRAFÍA

DICCIONARIO COMBINATORIO PARA

HABLANTES DE PORTUGUÉS

Rebeca Martínez Aguirre

Bosque Muñoz, I. (2011). Deducing collocations, en I. Boguslavsky y L. Wanner. (Eds.), Proceedings of the 5th International Conference on Meaning-Text Theory. Barcelona: Servicio de Publicaciones de la Universidad Pompeu Fabra, pp VI-XXIII. Recuperado de http://olst.ling.umontreal.ca/pdf/proceedingsMTT2011.pdf

Bosque Muñoz, I. (2017). On the conceptual bases of collocations. Restricted adverbs and lexical selection. En S. Torner y E. Bernal. (Eds.), Collocations and other lexical combinations in Spanish: theoretical, lexicographical and applied perspectives, Londres/Nueva York: Routledge, pp. 9-20.

Calderón Campos, M. (1994). Sobre la elaboración de diccionarios monolingües de producción: las definiciones, los ejemplosy las colocaciones léxicas, Granada: Universidad de Granada.

Castillo Carballo, M. A. (2002). El aspecto colocacional en la producción lexicográfica, en J. I. Pérez y M. Campos. (Eds.), Cuestiones de lexicografía (pp. 97-105), Lugo: Tris Tram.

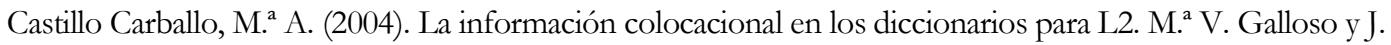
Prado. (Coords.), Diccionario, léxico y cultura, Huelva: Universidad de Huelva, pp. 71-83.

Castillo Carballo, M. ${ }^{a}$ A. (2015). Combinatoria léxica y lexicografía didáctica monolingüe. Estudios de Lexicografia, 1, pp. 74-80.

Climent de Benito, J. (2008). Los diccionarios semibilingües: principios y clasificación desde un punto de vista didáctico, Alicante: Biblioteca Virtual Miguel de Cervantes. Recuperado de http://www.cervantesvirtual.com/obra/los-diccionarios-semibilinges-principios-y-clasificacindesde-un-punto-de-vista-didctico-0/

Corpas Pastor, G. (1996). Manual de fraseología española, Madrid: Gredos.

Corpas Pastor, G. (2017). Collocations in e-bilingual dictionaries, en S. Torner y E. Bernal. (Eds.), Collocations and Other Lexical Combinations in Spanish. Londres/Nueva York: Routledge, pp. 173-199.

Cowie, A. P., (2002 [1999]). English Dictionaries for Foreign Learners: a History, Oxford: Oxford University Press.

Firth, J. R., (1968 [1957]). Papers in linguistics 1934-1951, Oxford: Oxford University Press.

Higueras García, M. (2004). Necesidad de un diccionario de colocaciones para aprendientes de ELE, en M. ${ }^{a}$ A. Carballo et al. (Coords.), Las gramáticas y los diccionarios en la enseñanza del español como segunda lengua: deseo y realidad. Actas del XV Congreso Internacional ASELE, Sevilla: Universidad de Sevilla, pp. 480490.

Higueras García, M. (2007). Estudio de las colocaciones léxicas y su enseñanza en español como lengua extranjera, Málaga/Madrid: ASELE/Ministerio de Educación y Ciencia.

Higueras García, M. (2017). Pedagocical principles for the teaching of collocations in the foreign language classroom, en S. Torner y E. Bernal. (Eds.), Collocations and other lexical combinations in Spanish: theoretical, lexicographical and applied perspectives (pp. 250-266). Londres/Nueva York: Routledge. 
SECCIÓN: LEXICOGRAFÍA

DICCIONARIO COMBINATORIO PARA

HABLANTES DE PORTUGUÉS

Rebeca Martínez Aguirre

Instituto Cervantes, (2007 [2006]). Plan Curricular del Instituto Cervantes Niveles de referencia para el español: B1-B2, Madrid: Instituto Cervantes/Biblioteca Nueva.

Koike, K. (2001). Colocaciones léxicas en el español actual: estudio formaly léxico-semántico, Alcalá de Henares: Servicio de Publicaciones de la Universidad de Alcalá de Henares.

Lewis, M. (1993). The Lexical Approach, Londres: Language Teaching Publications.

Maldonado González, C. (2017). Word combinations in general dictionaries, en S. Torner y E. Bernal (Eds.), Collocations and Other Lexical Combinations in Spanish (pp. 139-156). Londres/Nueva York: Routledge.

Martínez Aguirre, R. (2017). Las colocaciones léxicas en el diccionario bilingüe de uso españolportugués, português-espanhol: inclusión y tratamiento. Revista Virtual de Estudos da Linguagem. Recuperado de http://www.revel.inf.br/es/edicoes/?id=47

Martínez Navarro, M. ${ }^{a}$ R. (2005). El diccionario de L2 que «deseamos», en M. ${ }^{a}$ A. Castillo (Coord.), Las gramáticas y los diccionarios en la enseñanza del español como segunda lengua, deseo y realidad: Actas del XV Congreso Internacional de ASELE, Sevilla, España: Universidad de Sevilla, pp. 573-579

Mel'čuk, I. (1995). Phrasemes in Language and Phraseology in Linguistics, en, M. Everaert, E. van der Linden, A. Schenk y R. Schreuder (Eds.), Idioms: Structural and psychological perspectives (pp. 167-232), Hillsdale: Lawrence Erlbaum.

Mel'čuk, I.; Clas, A. y Polguère, A. (1995). Introduction à la lexicologie explicative et combinatoire, París/Louvain-laNeuve: Duculot.

Mitchell, T. (1971). Linguistic 'Goings-On': Collocations and Other Lexical Matters Arising on the Linguistic Record. Archivum Linguisticum, 2, pp. 35-69.

Molina Díaz, F. (2010). El tratamiento de las colocaciones en los diccionarios monolingües de español L2, en S. Ruhstaller y M. ${ }^{a}$ D. Gordón (Eds.), Diccionario y aprendizaje del español, Berna, Suiza: Peter Lang, pp. 213-232

Moreno, F. y Maia, N. (2003). Diccionario Bilingüe de Uso Español-Portugués, Português-Espanhol, Madrid: Arco Libros.

Nation, I. S. P. (1990). Teaching and learning vocabulary, New York: Newbury House.

Nation, I. S. P. (2001). Learning vocabulary in another language, Cambridge: Cambridge University Press.

Nesselhauf, N. (2004). What are collocations?, en D. J. Allerton, N. Nesselhauf y P. Skandera. (Eds.), Phraseological Units: basic concepts and their application, Basel, Suiza: Schwabe Verlag. pp. 1-21.

Perdiguero Villarreal, H. (2010). Logros y retos de los diccionarios de español para extranjeros: noticias halagüeñas, en S. Ruhstaller y M. ${ }^{a}$ D. Gordón (Coords.), Diccionario y aprendizaje del español, Berna: Peter Lang, pp. 133-154. 
Pérez Basanta, C. (1999). La enseñanza del vocabulario desde una perspectiva lingüística y pedagógica, en M. ${ }^{a}$ S. Salaberri. (Ed.), Lingüística aplicada a la enseñanza de lenguas extranjeras, Almería: Universidad de Almería, pp. 262-306.

Prado Aragonés, J. (2005a). El uso del diccionario para la enseñanza de la lengua: consideraciones metodológicas. Revista Artes y Letras. XXIX (Especial), pp. 19-28.

Prado Aragonés, J. (2005b). Tópicos culturales en los diccionarios de enseñanza de ELE, en M. ${ }^{a}$ A. Castillo. (Coord.), Las gramáticas y los diccionarios en la enseñanza del español como segunda lengua, deseo y realidad: Actas del XV Congreso Internacional de ASELE, Sevilla: Universidad de Sevilla, pp. 705-709.

Romero Aguilera, L. (2008). Colocaciones léxicas en diccionarios generales monolingües del español, en J. A. DeCesaris y E. Bernal (Coords.), Proceedings of the XIII EUR ALEX International Congress, Barcelona: Universitat Pompeu Fabra, pp. 1401-1408.

Ruiz Martínez, A. M.a (2007). La noción de colocación en las partes introductorias de algunos diccionarios monolingües del español. Revista de lexicografia, 13, pp. 139-182.

Tarp, S. (2006). Lexicografía de aprendizaje. Cadernos de Tradução, 2/18, pp. 295-317. 